\title{
Emergent Semiotics in Genetic Programming and the Self-Adaptive Semantic Crossover
}

\author{
Rafael Inhasz and Julio Michael Stern
}

\begin{abstract}
We present SASC, Self-Adaptive Semantic Crossover, a new class of crossover operators for genetic programming. SASC operators are designed to induce the emergence and then preserve good building-blocks, using metacontrol techniques based on semantic compatibility measures. SASC performance is tested in a case study concerning the replication of investment funds.
\end{abstract}

\section{Introduction}

Genetic Programming (GP) are evolutionary algorithms that work on populations, whose individuals represent possible (viable) solutions to the optimization problem, see [2] and 8, 9]. The solution functions, code or programs defining an individual are its genotype, while the image, graph or output of these functions are the individual's phenotype. An adaptation, cost or fitness function, computed from an individual's phenotype, represents the objective function of the optimization problem.

GP are meta-heuristics based on some key functions and operators inspired on evolution theories for biological species. Reproduction operators generate new individuals, the children, from existing ones, their parent(s), hence expanding the population. Mutation operators act on single individuals, for asexual reproduction, while crossover operators act on pairs of individuals, for sexual reproduction. A mutation operation generates a random change in the parent's code. This change is usually small, but may have important consequences for the individual fitness, often bad, but sometimes good. A

Rafael Inhasz

Institute of Mathematics and Statistics, University of São Paulo, Brazil e-mail: rafael.inhasz@yahoo.com.br

Julio Michael Stern

Institute of Mathematics and Statistics, University of São Paulo, Brazil e-mail: jstern@ime.usp.br 
crossover operation generates new children by swapping portions of their parents' codes at randomly selected recombination points.

Reproduction operators are random operators. However, they only introduce a limited amount of entropy (noise or disorder) in the process, making it possible for children to inherited many characteristics coded by their parents' genotype. GP starts from an initial population, that may be randomly generated. The population then evolves according to the random reproduction and selection stochastic processes. The entropy introduced at reproduction allows for creative innovation, while the selection processes induce learning constraints. Under appropriate conditions, after many generations (near) optimal individuals are likely to emerge in the population.

The schemata theorem, arguably the most characteristic result of GP theory, shows that, under appropriate conditions, the emerging optimal solutions naturally exhibit a hierarchical modular organization. Such modules are known as genes, schemata or building blocks, see [6, 11, 17, 19, 23. In light of the Schemata theorem, it is easy to understand that efficient crossover operators must be compatible with, preserve, favor, or even induce the emerging modular structure. More efficient operators are less likely to break down existing building blocks during reproduction, an unfortunate event known in the literature as destructive crossover.

This paper presents a new crossover operator, named SASC or SelfAdaptive Semantic Crossover. SASC is based on meta-control techniques designed to guide the random selection of recombination points by a measure of semantic compatibility between the portions of code being swapped. It is important to realize that SASC's meta-control system is not hard-wired or pre-defined. On the contrary, it is an emerging feature, co-evolving with the population. The meta-control system is based on the history of each individual in the population. However, the required historical information, accumulated during the individual's evolutionary line, is very limited. Hence, its implementation only generates a minor computational overhead.

Section 2 gives a short review of genetic programming. Section 3 explains some meta-control concepts and defines SASC - the self-adaptive semantic crossover operator. Section 4 presents some ideas of semiotics and cognitive constructivism that inspired this line of research. Section 5 gives some implementation details and section 6 compares the performance of SASC and standard crossover operators at a case study concerning the replication of financial investment portfolios. Section 7 presents our conclusions and final remarks.

\section{Genetic Programming in Functional Trees}

In this and the following sections, we deal with GP in the context of functional trees. In this setting, the objective is to find the correct specification, the best functional form, or just a good emulation of a complex target function. The 


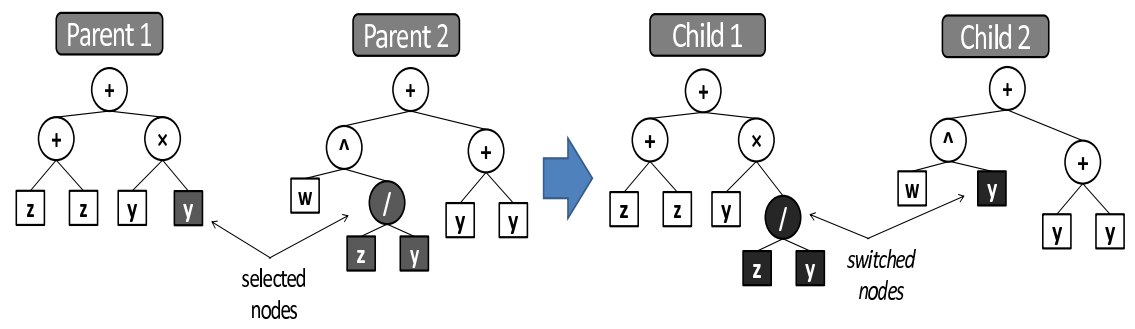

Fig. 1 Example of destructive crossover.

only information available about the target function is an input-output databank. An individual in the population is represented as a tree, with atoms at the leaves representing constants or input variables, and primitive operators at internal nodes. The root node output, at the top of the tree, expresses the individual's phenotype. Atoms and primitive operators are taken from finite sets, $A=\left\{a_{1}, a_{2}, \ldots\right\}$ and $O P=\left\{o p_{1}, o p_{2}, \ldots o p_{p}\right\}$. Each operator, $o p_{k}$, takes a specific number of arguments, $r(k)$, known as the arity of $o p_{k}$.

Figure 1 shows four individuals in the population of a GP trying to emulate the target function, $f(w, y, z)=y^{2}+w^{z / y}$, from the primitive set of expanded arithmetic operators, $O P=\{+,-, \times, /, \wedge\}$. Inputs at the leaves are represented in a square, and operators at internal nodes or at the root are represented in a circle. Figure 1 also shows a crossover, having the first two individuals as parents and the last two as children. The recombination points in the parent trees are highlighted. Notice that the first parent contains the component, partial solution or building block for the first term in the target function, $y^{2}$, while the second parent contains the building block for the second term, $w^{z / y}$. Since none of these interesting building blocks are preserved in the children, we call this a destructive crossover. A child inherits its root node, and hence usually most of its code, from the parent we call its mother, while from its father the child receives a, usually smaller, sub-tree. Hence, in this example, parent 1 and 2 are, respectively, mother and father of child 1, and father and mother of child 2 .

Angeline, 1], proposed the SSAC - Selective Self-Adaptive Crossover - in order to make destructive crossovers less likely. Standard crossover selects recombination points in a parent tree with uniform distribution.

In SSAC like crossovers, each node, $n(i)$, stores a meta-control variable, $\rho_{i}$, a real number bounded to the normalization constraint: $0 \leq \rho_{\min } \leq \rho_{i} \leq$ $\rho_{\max }$. The probability of selecting node $n(i)$ for recombination is proportional to $\rho_{i}$. That is, the probability of choosing node $n(i)$ as the recombination point in that tree is $p_{i}=\rho_{i} / \sum_{j} \rho_{j}$.

After a crossover, nodes at the children, carry along the meta-control variables they had at the parents, and afterwards suffer the effect of random noise. For example, the meta-control variable in node $n(i)$ can be updated as $\rho^{\prime}{ }_{i}=\left(1+\mu_{i}+\sigma_{i} \epsilon\right) \rho_{i}$, where $\epsilon$ is the standard Normal random variable, $\mu_{i}$ is 
a zero or positive drift, and $\sigma_{i}$ is a positive scale factor. All $\rho_{i}$ are initialized at the minimum value, $\rho_{\text {min }}$, and allowed to move inside the normalization bounds. For details on Angeline's original implementation, see 11. Several interesting variations can be found in the proceedings in the reference list.

The intuition behind SSAC is that survivors in the GP competition process are well adapted individuals, containing good building blocks. Moreover, successful breeders must be able to give these building blocks intact to their children. At these breeders, large meta-control variables should mark plausible building blocks, indicating good recombination points to be used (again) in the future. Genotype codes and meta-control variables should both coevolve, facilitating the emergence, marking, and preservation of good building blocks.

Angeline [1] also presents an alternative method, SAMC - Self-Adaptive Multi-Crossover, where the meta-control variables can be interpreted as absolute probabilities, that is, $\rho_{\max }=1$. SAMC selects a recombination point in a two step process: First, all nodes in the tree receive a Boolean mark, 1 with probability $\rho_{i}$, and 0 otherwise. At the second step, the recombination point is selected from the nodes marked 1 with uniform distribution.

Before ending this section we make some additional comments about the schemata theorem. As already mentioned in the introduction, it is in the light of the schemata theorem that we can understand why efficient crossover operators must be compatible with, preserve, favor, or even induce the emerging modular structure. However, Holland's original theorem was stated for a very particular case, namely, genetic algorithms using string coded programs. Schemata theories extend this fundamental result to genetic programming using functional trees, see [15, 16, 18. Hence, we must rely on Rosca, Poli and Langdon's results to keep our work on well founded theoretical ground.

\section{The Self-Adaptive Semantic Crossover}

SASC descends from Angeline's SSAC and SAMC operators, but it also incorporates information concerning the sub-trees rooted at the nodes in possible recombination points. The first information used for this purpose is captured through the notion of similarity. (Sub)Trees $A$ and $B$ are phenotypically similar if their output, computed at the records available on the data bank, agree within a specified tolerance.

We assume that two parents, father $A$ and mother $B$, have been selected for crossover according to the mating distributions used at the GP. SASC starts by using a first heuristic procedure to define new meta-control variables, $\delta_{i}$, at the nodes, $n(i)$, of the father, $A$. Let $A(i)$ be the sub-tree of $A$ rooted at $n(i)$. For each sub-tree, $A(i)$, the procedure searches the mother, $B$, for sub-trees, $B(j)$, that are similar to and also either the same size or shorter than $A(i)$. If such a short similar sub-tree is found, $\delta_{i}=\rho_{\text {min }}$. Otherwise, $\delta_{i}=\rho_{i}$. Finally, the recombination point at the father is randomly selected 
with probabilities $p_{i}=\delta_{i} / \sum_{j} \delta_{j}$. The intuition behind the first heuristic procedure is to stimulate innovation, that is, to only chose recombination points at the father that, by the crossover operation, are able to contribute with an innovative component, $A(i)$, that is not already present in the mother or, at least, to contribute with a similar component that is more efficiently coded.

After the recombination point at the father, $n(i)$ - root of sub-tree $A(i)$, has been chosen, a second heuristic procedure selects the recombination point at the mother, $m(j)$ - root of sub-tree $B(j)$. Again, new meta-control variables, $\lambda_{j}$ are defined for the nodes $m(j)$, followed by a random selection with probabilities $p_{j}=\lambda_{i} / \sum_{j} \lambda_{j}$. The idea behind this second heuristic procedure is to stimulate the crossover to exchange sub-trees, $A(i)$ and $B(j)$, with analogous meanings, compatible semantics, similar interpretations, etc. This heuristic procedure draws inspiration from biology, where analogy is defined as compatibility in function but not necessarily in structure or evolutionary origin.

The formal expression used to evaluate the meta-control variables at the second heuristic procedure is:

$$
\lambda_{j}=w_{0}+\left[\sum_{d=1}^{D} w_{d} C_{k}(A(i), B(j))\right]
$$

The index $d$ spans $D$ semantic dimensions or factors. The positive weights, $w_{d}$, add to one, and the semantic compatibility measures, $C_{k}$, are normalized in the interval $[0,1]$.

The functional form of the compatibility measures, $C_{k}($ ), are completely dependent on insights and interpretations for the actual problem being solved. In the case of the arithmetic functional tree presented at this section, the analogy between two sub-trees could be established, for example, simply by the fraction of input variables they share in common. In this case, blocks coding $y^{2}$ e $2 y$ would have compatibility measure equal to 1 , while the blocks coding $y^{2}$ and $w^{z / y}$ would have compatibility measure equal to $1 / 3$.

After a SASC crossover, the children's nodes carry along the meta-control variables, $\rho_{i}$, they had at the parents, and are afterwards updated by a random perturbation. We used a standard Normal multiplicative noise with drift $\mu_{i}$ and scale factor $\sigma_{i}$, that is, $\rho_{i}^{\prime}=\left(1+\mu_{i}+\sigma_{i} \epsilon\right) \rho_{i}$. At practical implementations we always used a positive drift at the recombination points, and a null drifts elsewhere. Sometimes we also used scale factors, $\sigma_{i}$, that decrease with the height of node $n(i)$. For instance, take $\sigma_{i}$ inversely proportional to the depth of sub-tree $A(i)$. Using larger scale factors at lower nodes can help to induce the emergence of smaller building-blocks, that are more efficiently coded, and less prone to destructive crossover. 


\section{Emerging Building Blocks and Semiotics}

In the following sections we explain our implementation of SASC methods, present an application case, and gauge its performance. However, before proceeding to finer details, this section tries to provide a larger picture, presenting the general framework that lead us to this line of research and some of the intuitions that inspired the name and definition of the SASC operator. Those readers interested mainly in the algorithmic aspects of the SASC operator can skip this section without prejudice. Nevertheless, the ideas presented in this section may provide a conceptual framework to examine similar algorithms and, in so doing, encourage or influence future research.

As stated in the introduction, under appropriate conditions, evolutionary systems naturally exhibit a hierarchical modular organization. The spontaneous emergence of hierarchical modular structures in natural or artificial evolving organisms is further studied in [19] and [23-25].

At the same time, the need or willingness to understand such systems leads to the attribution of meanings or interpretations corresponding to the systems' constituent parts. Specially in the case of inferential systems, this attribution of meaning leads, in turn, to consider the semiotic character of such parts. In this condition, we consider the system's modules as symbols representing concrete referents, that is, signs pointing to real things existing in the world or truthful relations occurring in the systems' environment. In this setting, rules of composition or coherent organization for the system's modular structures can be considered as articulation rules for terms in a systemic language. Hence, in this perspective, the emergence of a hierarchical modular organization in an evolutive inferencial system corresponds to a process of linguistic ontogenesis.

In the epistemological framework of cognitive constructivism, the semiotic character and the consequent semantic interpretation of the system's modular components arises from the inherent complementarity of a dual perspective:

(1) In the autopoietc system perspective, these components are seen as building blocks of an autopoietic unit, structured in a hierarchical and modular organization.

(2) In the reasoning model perspective, the same components are seen as constituent parts of a reasoning system, like sub-routines of a complex code, functions of a large program, etc.

The semiotic character and semantic interpretations of building blocks correspond to coherent and consistent (although possibly multimodal) forms in which these modules are used as operational tools, instrumental agents, partial production units, etc. used to implement solutions for the problems faced by the autopoietic system interacting with its environment.

The motivating and validating argument for the superposition of these two distinct and complementary views of the emerging structures, namely, that of an autopoietic system and that of a reasoning model, is given by Humberto 
Maturana and Francisco Varela celebrated principle stating that - every autopoietic system is an inferential system, and its domain of interactions a cognitive domain. This principle is stated in [13. p. 10], and it is further explored, within the epistemological framework of cognitive constructivism, in [4] and [20-23].

The ideas briefly discussed in this section are far to general and abstract to directly generate specific algorithms or formulate explicit modeling solutions. Nevertheless, we hope that these ideas can be useful to investigate new techniques bearing some similarity to the specific methods presented in this article. If so, these ideas could be helpful in the "downward" direction leading to the development of new heuristic techniques used to accelerate the convergence or to induce the emergence of meaningful components in the underlying evolutive process.

At the same time, it is also true that the precise meaning of concepts used in a given epistemological framework can only be fully accessed analyzing specific theories, well defined hypothesis, concrete models or existing embodied system. Hence, we hope that the ideas discussed in this section can also be useful in the "upward" direction, fostering further research in the fields of applied semiotics, cognitive constructivism, and epistemological aspects of inferential systems.

\section{Implementation}

Our implementation of SASC methods is based on ECJ, an open-source evolutionary computing system written in Java. ECJ is developed at George Mason University's ECLab Evolutionary Computation Laboratory. ECJ maintains a well organized object-oriented design. Its powerful classes and methods proved to be very flexible, and could be easily extended to our purposes. The SASC package, developed by the first author, extends some ECJ classes in order to easily implement the methods under discussion. Most of the new code is concentrated at the class $S A S C N$ ode, used to represent functional trees evolving by SASC GP. This class also includes abstract methods that facilitate the implementation of semantic compatibility measures, specified at sub-classes implemented for each specific problem.

Finally we should mention that ECJ supports distributed computing, specifying the desired number of parallel threads as a parameter to be set according to the available resources offered by the hardware and operating system. This feature was especially useful for multi-population scenarios, to be described in the next section, where SASC GP had an excellent performance.

\section{Case Study}

SASC operator was compared to standard crossover operators at a test case problem concerning the replication of an hypothetical investment fund. 
Although hypothetical, this problem has strong similarities with real problems regarding the construction of synthetic portfolios faced by the first author in his professional activities. Portfolios of this kind are typical of correlation trade, since its return statistics are sensitive to the correlation matrix for the returns of various components in a basket. Such portfolios can be easily synthesized using readily available exotic derivatives like rainbow options, that is, calls or puts on the best or worst of several underlying assets.

Lemon, the hypothetic fund, is based on stocks negotiated at BMESFBovespa - São Paulo Securities, Commodities and Futures Exchange. Lemon's daily log-return, $r_{t}$, is given by the log-return average of four components, $r_{t}^{k}$, corresponding to key economic sectors. These are, using standard BMEFBovespa equity codes:

$$
\begin{aligned}
& r^{1}=\min (B B D C 4, \text { PETR } 4, B B A S 3), \\
& r^{2}=\min (L A M E 4, L R E N 3, N E T C 4), \\
& r^{3}=\max (T N L P 4, T C L S 4, V I V O 4) \text { and } \\
& r^{4}=\max (C Y R E 3, \text { ALLL11, GFSA4). }
\end{aligned}
$$

These components represent four key economic sectors: Telecommunications, construction and transports, finance and cyclic consumption.

An asset manager wants to synthesize a second fund, Lime, with the objective of tracking fund Lemon. However, only the daily share values of fund Lemon are available, not its operational rules. Of course, GP was the method chosen to find the best specification of the synthetic portfolio Lime. The atoms for this problem are the log-returns of 63 of the most liquid stocks negotiated at BMEFF-Bovespa, that include all the stocks used to specify fund Lemon. The primitive operators are $\{\max , \min , \operatorname{mean}\}$, the maximum, minimum and mean value of two real numbers. The training data bank consists of the daily log-returns of fund Lemon and all 63 stocks, computed from 04-Nov-2008 to 01-Apr-2009.

The fitness function for this problem is the mean squared error between the synthetic and the target log-returns, plus a regularization term adding, for each node, $n(i)$, a penalty $\pi(i)$. For the application at hand, we used $\pi(i)=c_{h(i)} 2^{h(i)-1}$, where $h(i)$ is the height of node $n(i)$. For the example at hand, we used $c_{h(i)}=1$ at the root node and zero otherwise. The purpose of regularization term is to avoid needless complexity and over-fitting in the final model, see [5].

In the GP experiments, we used two distinct population scenarios. Scenario 1: One population of 300 individuals evolving over 700 generations, Scenario 2: 8 populations of 300 individuals each, that first evolve in isolation over 400 generations and are then allowed to merge and evolve for 100 generations more. In both scenarios the GP is allowed to warm-up using the standard crossover, 200 generations for scenario 1 and 100 for scenario 2, and then switch to (or not) to SASC crossover. SASC's semantic compatibility function is the Boolean indicator of having at least one atom in common. 
The actual GP implementation uses a dual tree representation for each individual in the population, as suggested in Angeline original paper, 1]. The first tree only stores the genotype used to code the function expressed by the individual's phenotype. Meanwhile, the second tree only stores meta-control variables.

GP meta-parameters were set as follows: mutation rate was set at $5 \%$, using a 3-round tournament selection process. Crossover rate was set at $95 \%$, using a 7-round high pressure / 3-round low pressure combination of father / mother selection, see 23. $\rho_{\min }=0.001, \rho_{\max }=0.999, w_{0}=0.01, w_{1}=0.99$, $\sigma_{i}=0.4$ for $h(i)=2$ and approximately inversely proportional to the node height for $h(i)>2$. Further details about the algorithm fine tuning can be seen at the source code documentation, available from the first author.

Figure 2 compares the GP results using standard and SASC crossover operators. The use of Angeline's original SSAC instead of the standard crossover operator had only a minor impact in GP performance, and is not shown in the figure. This figure displays $95 \%$ confidence intervals for the mean square error of the best solution found over 50 independent GP runs.

Figure 3 shows the best empirical solution found by SASC GP. The figure also highlights the building blocks encapsulated by meta-control variables larger than a critical threshold. This solution replicates very well the target fund. Notice that each of the highlighted building blocks corresponds to one of the key economic sectors used to define the operation rules of fund Lemon.

Each best solution found at a batch of 50 SASC GP experiments under scenarios 1 and 2 was categorized according to the number of key economic sectors represented by a constituent building block. Table 1 displays the average mean square error of each category. This table shows that better adjusted
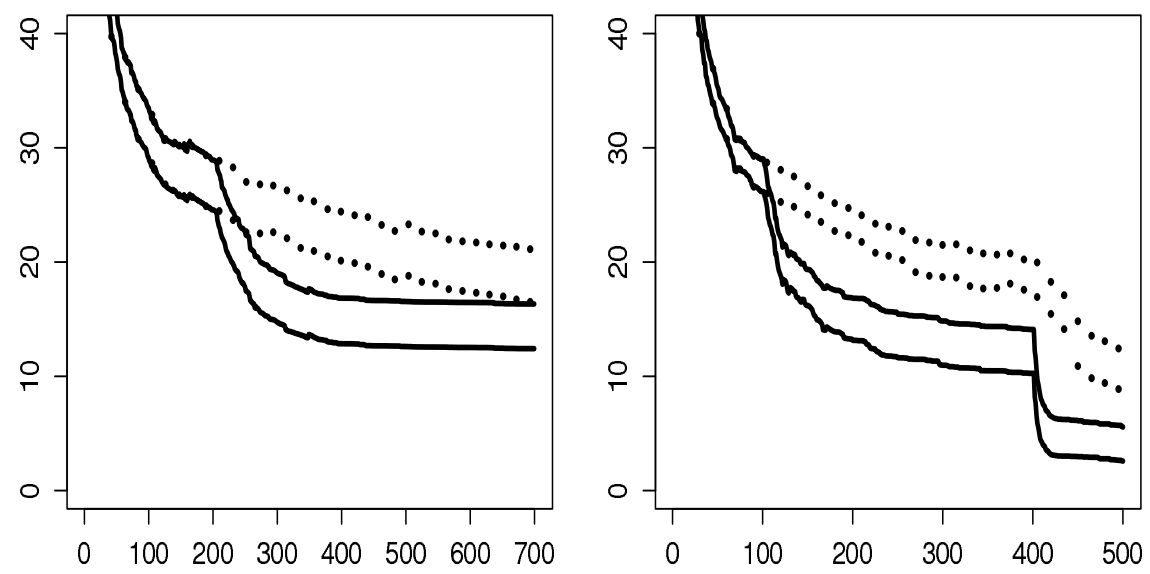

Fig. 2 Confidence interval for best solution MSE by generation. Crossovers' comparative performance: Standard $(\cdots)$ and SASC $(-)$. 


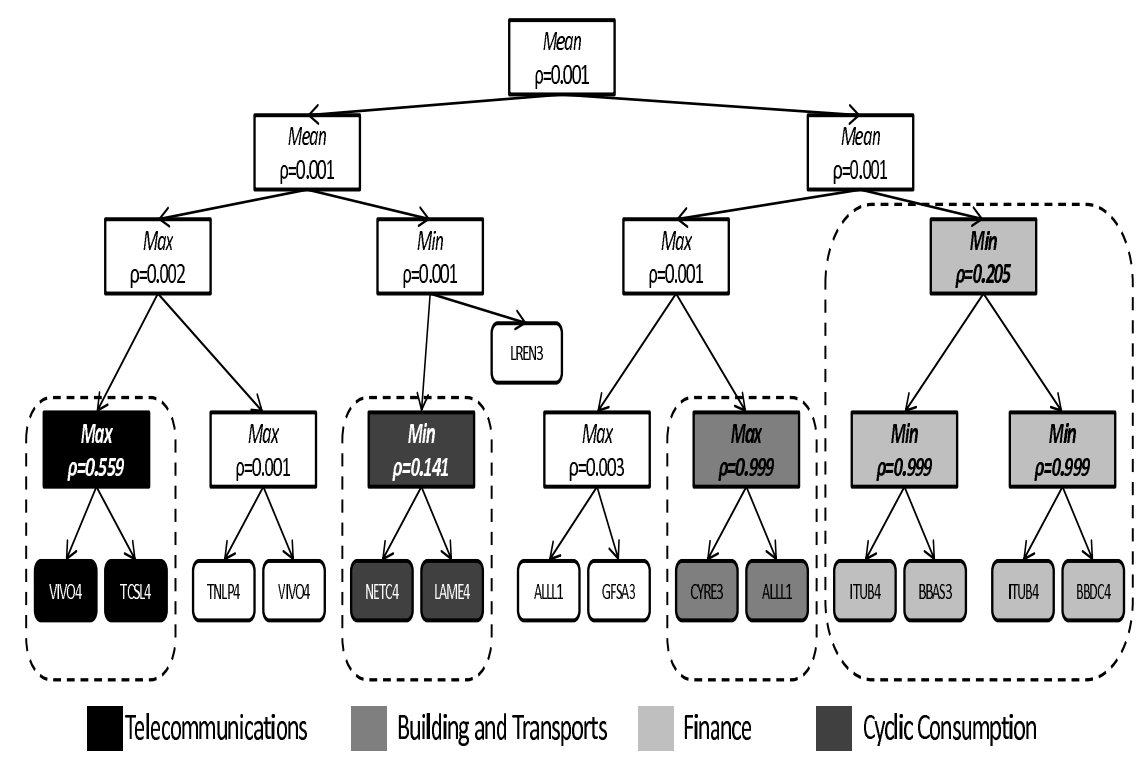

Fig. 3 Emerging building-blocks in near-optimal solution.

Table 1 Number of key economic sectors represented by building blocks

\begin{tabular}{lcccc}
\hline Category & Scenario & MSE & Scenario & MSE \\
\hline One key sector & $14 \%$ & 12.3 & $10 \%$ & 8.9 \\
Two key sectors & $16 \%$ & 8.1 & $30 \%$ & 1.9 \\
Three key sectors & $8 \%$ & 9.3 & $38 \%$ & 1.4 \\
Four key sectors & $0 \%$ & - & $4 \%$ & 0.1 \\
Other (spurious) blocks & $62 \%$ & 21.7 & $18 \%$ & 10.2 \\
\hline
\end{tabular}

functional trees have more of the four key economic sectors present as a building block. This conclusion may be obvious to someone knowing the operating rules of Lemon, the original target fund. However, it is remarkable that the best solutions offered by SASC GP for the replication fund Lime, synthesized only from input-output data, are able to capture so well the logic and semantics of fund Lemon.

\section{Conclusions and Final Remarks}

From Figure 2, we can conclude that, at least for the test case at hand, GP has a much better performance when using SASC than the standard crossover operator. At scenario 2 the best empirical solution, shown at Figure 3, is found repeatedly. At scenario 1, SASC not only achieves better results, but 
also seems to greatly accelerate the finding of good solutions. These effects are even stronger at scenario 2, where a second acceleration effect is clear just after the populations merge. At this final stage, one can observe that the best solution are formed purging spurious building blocks and combining good building blocks that had emerged at the previously isolated populations. It is as if SASC were able to isolate, identify, and collect good building blocks.

The explanatory power of the emergent building blocks, that is, on one hand, how well they capture the semantics of the system under study and, on the other hand, how much they contribute to its prediction accuracy, is made even clearer by Table 1. Accordingly, Figure 3 suggests that SASC GP can also provide an implicit method of semantic analysis. That is, at least in our case study, the internal operational logic and the semantics of the target system is adequately represented by the building blocks of the best solutions synthesized by SASC GP. Nevetheless, it is important to keep in mind that these logical and semantic relations were not externally imposed or driven, but are truly emergent properties co-evolving with the GP solutions.

\section{Future Research}

In future research we plan to investigate techniques of self-adaptive metacontrol using abstract type node labels as auxiliary control variables. Transformation rules for label mutation and label compatibility rules for permissible recombination points should be able to induce building block formation and encapsulation, and also be able to foster emergent semantic interpretations, even in problems lacking natural heuristics for explicit semantic compatibility measures.

Acknowledgements. The authors are grateful for the support of IME-USP, The Institute of Mathematics and Statistics of the University of São Paulo, FAPESP, Fundo de Amparo à Pesquisa do Estado de São Paulo, CNPq, The Brazilian National Research Council, and BMEFF-Bovespa, The São Paulo Securities, Commodities and Futures Exchange. The authors are also grateful for the helpful comments of Marcelo Lauretto and an anonymous referee.

\section{References}

1. Angeline, P.: Two self-adaptive crossover operators for genetic programming. In: Angeline, P.J., Kinnear, K.E. (eds.) Advances in Genetic Programming. Complex Adaptive Systems, vol. 2, ch.5, pp. 89-110. MIT Press, Cambridge (1996)

2. Banzhaf, W., Francone, E.D., Keller, R.E., Nordin, P.: Genetic Programming, an Introduction: On the Automatic Evolution of Computer Programs and its Applications. Morgan Kaufmann, San Francisco (1998)

3. Banzhaf, W., Poli, R., Schoenauer, M., Fogarty, T.C. (eds.): EuroGP 1998. LNCS, vol. 1391. Springer, Heidelberg (1998) 
4. Borges, W., Stern, J.M.: The rules of logic composition for the bayesian epistemic e-values. Logic Journal of the IGPL 15(5-6), 401-420 (2007)

5. Cherkaasky, V., Mulier, F.: Learning from Data. Wiley, NY (1998)

6. Holland, J.H.: Adaptation in Natural and Artificial Systems. University of Michigan Press, Ann Arbor (1975)

7. Iba, H., Sato, T.: Meta-level strategy for genetic algorithms based on structured representation. In: Proc. of the Second Pacific Rim International Conference on Artificial Intelligence, pp. 548-554 (1992)

8. Koza, J.R.: Genetic programming: On the programming of computers by means of natural selection. MIT Press, Cambridge (1992)

9. Koza, J.R.: Genetic programming II: automatic discovery of reusable programs. MIT Press, Cambridge (1994)

10. Koza, J.R., Deb, K., Dorigo, M., Fogel, D.B., Garzon, M., Iba, H., Riolo, R.L. (eds.): Genetic Programming 1997: Proceedings of the Second Annual Conference. Morgan Kaufmann, San Francisco (1998)

11. Langdon, W.B., Poli, R.: Foundations of Genetic Programming. Springer, Heidelberg (2002)

12. Lauretto, M., Nakano, F., Pereira, C.A.B., Stern, J.M.: Hierarchical forecasting with polynomial nets. In: [14], p. 305-315 (2009)

13. Maturana, H.R., Varela, F.J.: Autopoiesis and Cognition. The Realization of the Living. Reidel, Dordrecht (1980)

14. Nakamatsu, K., Phillips-Wren, G., Jain, L.C., Howlett, R.J. (eds.): New Advances in Intelligent Decision Technologies. Springer, Heidelberg (2009)

15. Poli, R., Langdon, W.B.: A new schema theory for genetic programming with one-point crossover and point mutation. In: [10], pp. 278-285 (1997)

16. Poli, R., Langdon, W.B.: A review of theoretical and experimental results on schemata in genetic programming. In: [3], pp. 1-15 (1998)

17. Reeves, C.R.: Modern Heuristics for Combinatorial Problems. Blackwell Scientific, Malden (1993)

18. Rosca, J.P.: Analysis of complexity drift in genetic programming. In: [10], pp. 286-294 (1997)

19. Simon, H.A.: The Sciences of the Artificial. MIT Press, Cambridge (1996)

20. Stern, J.M.: Cognitive constructivism, eigen-solutions, and Sharp statistical hypotheses. Cybernetics and Human Knowing 14(1), 9-36 (2007a)

21. Stern, J.M.: Language and the self-reference paradox. Cybernetics and Human Knowing 14(4), 71-92 (2007b)

22. Stern, J.M.: Decoupling, sparsity, randomization, and objective bayesian inference. Cybernetics and Human Knowing 15(2), 49-68 (2008a)

23. Stern, J.M.: Cognitive Constructivism and the Epistemic Significance of Sharp Statistical Hypotheses. In: Tutorial book for MaxEnt 2008, The 28th International Workshop on Bayesian Inference and Maximum Entropy Methods in Science and Engineering, Boracéia, São Paulo, Brazil, July 6-11 (2008b)

24. Stern, J.M., Colla, E.C.: Factorization of sparse bayesian networks. In: [14], pp. 275-294 (2009)

25. Stern, J.M.: The Living and Intelligent Universe. Tech.Rep. MAP-IME-USP2009-04. Presented at MBR-09, Campinas, Brazil (2009) 